\title{
Un acercamiento al concepto de representación: el caso de las mujeres políticas en el estado de Chihuahua
}

\section{An approach to concept of representation: the case of women politicians in the state of Chihuahua}

\section{Martha Estela Pérez* \\ María Isabel Escalona Rodriguez}

*Nacionalidad: Mexicana. Grado: Doctorado en Ciencias Sociales. Especialidad en relaciones de poder y cultura política. Especialización: Estudios de Género. Mujeres, movimientos sociales y participación política. Adscripción: Profesora-Investigadora de la Universidad Autónoma de Ciudad Juárez. Correo electrónico: meperez@uacj.mx

**Nacionalidad: Mexicana. Grado: Estudiante del Programa de Doctorado en Ciencias Sociales. Especialización: Gobierno y Políticas Públicas. Adscripción: Universidad Autónoma de Ciudad Juárez. Correo electrónico: al116176@alumnos.uacj.mx

Fecha de recepción: 11 de septiembre de 2012

Fecha de aceptación: 01 de noviembre de 2012 


\section{Resumen}

En este documento se reflexiona sobre el fenómeno de la representación simbólica y política de las mujeres en el estado de Chihuahua. Este caso se centra en la escasa representación del sexo femenino en la política institucional y, de igual forma, se hace un acercamiento a los esquemas culturales que siguen vigentes en la esfera pública, los cuales no sólo inciden en el quehacer político femenino y masculino, sino que también conllevan a generar espacios de discriminación e inequidad y obstaculizan el empoderamiento femenino.

Palabras clave: representación politica, representación simbólica, inequidad, discriminación, politica, empoderamiento

\section{Abstract}

This paper reflects on the phenomenon of political and symbolic representation of women in the state of Chihuahua. This case focuses on the low representation of females in the institutional policy and similarly, it is an approach to cultural patterns that are still alive in the public sphere, which not only affect the male and female political activity, also lead to create opportunities for discrimination and inequality and hinder women's empowerment.

Key words: political representation, symbolic representation, inequality, discrimination, political empowerment 


\section{Introducción}

El acceso del sexo femenino al poder puede variar según el contexto sociocultural de cada país, en el caso específico de México, existen prácticas que crean espacios de inequidad para las mujeres causadas por ideas preconcebidas sobre la separación de lo público-privado, lo cual tiene incidencia directa en la contienda por los puestos de representación popular. Es un hecho que hoy día, a pesar de la creación de prerrogativas que buscan incidir positivamente en las brechas de desigualdad, como es la ley de cuotas, siguen siendo relegadas no sólo de ciertos cargos, sino de los de mayor jerarquía política.

Hasta ahora, México no ha sido gobernado por una mujer, como en otros países de América Latina. ${ }^{1}$ En nuestro país, concretamente en el poder Ejecutivo que se elige mediante el sufragio, han surgido sólo cuatro candidatas a la presidencia de la República ${ }^{2}$ y en la pasada contienda electoral (2012) otra mujer, Josefina Vázquez Mota, fue la candidata del Partido Acción Nacional (PAN), uno de los tres partidos políticos con mayor presencia en México y que gobernó hasta 2012 cuando fue desplazado por el PRI en la presidencia de la República. Los otros tres candidatos fueron varones: Enrique Peña Nieto del Partido Revolucionario Institucional (PRI), Andrés Manuel López Obrador del Partido de la Revolución Democrática (PRD) y Gabriel Quadri del Partido Nueva Alianza (PANAL). ${ }^{3}$

1 Actualmente Argentina, Brasil y Costa Rica son gobernados por mujeres. En el pasado, Chile, Panamá, Ecuador, Nicaragua, Haití y Bolivia tuvieron una presidenta. Información disponible en http://www.losandes.com.ar/notas/2010/11/1/presidentasamerica-latina-524571.asp [consultado el 25 de marzo de 2012].

2 La primera mujer fue Rosario Ibarra de Piedra en 1982 por el Partido Revolucionario de los Trabajadores (PRT), en 1988 se postuló nuevamente por el Partido del Trabajo; la segunda mujer fue Cecilia Soto González por el Partido del Trabajo. La tercera mujer fue Marcela Lombardo Otero, quien participó por el Partido Popular Socialista en 1994. La cuarta mujer fue Patricia Mercado postulada por el Partido Alternativa Social Demócrata y Campesino en 2006. Información disponible en http://yopolitico. redpolitica.mx/notas/?p=1359 [consultado el 25 de marzo de 2012].

3 www.ife.org.mx [consultado el 29 de abril de 2012]. 
En el caso de las entidades federativas de nuestro país, seis han sido las mujeres que han gobernado algún estado de la República desde que se obtuvo el derecho al voto en 1953: Griselda Álvarez el estado de Colima, de 1979 a 1985; Beatriz Paredes Rangel el estado de Tlaxcala, de 1987 a 1992; Dulce María Saurí Riancho el estado de Yucatán, de 1991 a 1994; Rosario Robles Berlanga el Distrito Federal, de 1990 al 2000; Amalia García el estado de Zacatecas, de 2004 a 2010; y finalmente, Ivonne Ortega Pacheco el estado de Yucatán, de 2007 a $2013 .^{4}$

En lo que respecta al poder Legislativo, las mujeres han buscado espacios como representantes populares con una participación activa en los partidos políticos y es donde han tenido mayor presencia, aunque no suficiente. De hecho, han sido en las legislaturas donde se han hecho notar con más claridad los esfuerzos para modificar las leyes con el objetivo de incrementar su participación en posiciones de poder. Sin embargo, los números no son halagüeños: 124 escaños, es decir el $27.8 \%$ del total de 500 curules, ${ }^{5}$ pues el congreso federal constituido en 2009, si bien registró la cuota de mujeres más alta en la historia, constituyó sólo el 27.8\% del total de curules. ${ }^{6}$ En el caso del Congreso del Estado de Chihuahua constituido en 2010, tiene sólo el 21\% de mujeres. Con esto podemos observar que pese a los esfuerzos en creación de leyes y del trabajo de los grupos femeninos para tener mayor acceso a puestos públicos, los números no reflejan la labor que realizan para integrarse a los espacios institucionales.

El objetivo central en este documento es ofrecer un panorama general que nos explique a través del análisis de las opiniones, experiencias y trayectorias de algunas mujeres que participan en la práctica política institucional del estado de Chihuahua, la problemática de la

4 www.ife.org.mx [consultado el 29 de abril de 2012].

5 Información disponible en http://informacionpolitica.wordpress.com/category/legislatura/ [consultado el 29 de abril de 2012] y la página del Programa de Naciones Unidas para el Desarrollo (PNUD), www.undp.org.mx/spip.php?page=imprime\&id_article $=1758$ [consultado el 31 de octubre de 2012].

6 Información disponible en http://informacionpolitica.wordpress.com/category/legislatura/ [consultado el 29 de abril de 2012]. 
representación simbólica y política del sexo femenino, así como su falta de empoderamiento. La metodología utilizada corresponde a una investigación cualitativa realizada con entrevistas semi-estructuradas y observaciones de campo.

Los hallazgos que se revelaron en esta investigación señalan que existe inequidad, subrepresentación y falta de empoderamiento en el ámbito político chihuahuense, pues si bien las entrevistadas son mujeres que cuentan con privilegios porque han traspasado a espacios difíciles de llegar para otros ciudadanos(as), una vez que están ahí se encuentran con un ambiente que mantiene prácticas que no les permiten desarrollarse en igualdad de condiciones.

La primera parte de este documento analiza algunos fundamentos teóricos de género, la discriminación de las mujeres en el ámbito político y las distintas acepciones de representación. La segunda parte examina algunos datos que nos hablan de la situación de las mujeres chihuahuenses en la política, y finalmente se incluyen las conclusiones.

\section{I.- Género, mujeres y política}

El género es una representación corpórea que se integra por diversos elementos como son los símbolos y mitos culturales, la institucionalización y la organización social de las relaciones, el sistema de parentesco y la identidad subjetiva, los cuales se ven reflejados en doctrinas religiosas, educativas, científicas, legales, así como en la política (Lagarde, 1992; Scott, 1997).

Esta construcción social que se adjudica al sexo es una estructura conceptual estimulada por las normas que lo crean, lo consolidan y le otorgan una categoría, constituyéndose así un esquema que se convierte en un marco regulador y un proceso natural, el cual se asienta en la sociedad bajo ciertas significaciones sociales que modelan las interrelaciones jerarquizadas entre los géneros (Butler, 2001, 2002).

El concepto de género se puede entender como una condición construida y determinada en torno al cuerpo que se posee; como mujer o como varón se adquieren los significados, las experiencias y la 
carga de poder que los posiciona socialmente en los espacios públicos y privados. En este campo de relaciones quedan atrapados hombres y mujeres que viven y experimentan la construcción del género, el cual permea los ámbitos de interacción humana y el campo político no queda excluido de esta situación.

Los condicionantes del género se expresan en el espacio político a partir de las relaciones de poder, los papeles sexuados y los estereotipos sociales, los cuales a su vez se inscriben en esquemas de dominación y subordinación femenina, de manera que las mujeres transitan bajo reglas no escritas que dificultan su participación pública. Lo que aún las mantiene lejos, expresa Marta Lamas (2006), de constituirse como figuras de peso en el mundo político.

$\mathrm{El}$ origen de estas dificultades se fundamenta en la división sexual del trabajo que estableció una separación de actividades entre lo femenino y lo masculino, lo cual dejó simbolizada la separación entre lo público y lo privado-doméstico (Lamas, 2006), quedando el ámbito político de difícil alcance y desarrollo para las mujeres, al inscribirse como un dominio masculino. Sin embargo, trascender esta dicotomía publico-privado significa trascender también la relación producciónreproducción, es decir, reconstruir de manera distinta los determinismos biológicos, los roles, las actividades y los esquemas de pensamiento. De ahí que es necesario transformar los simbolismos que se fijan en el imaginario para reposicionar lo considerado como femenino y permitir una exitosa vinculación con el espacio político. ¿Pero, cómo esto sería posible? Una respuesta la podríamos encontrar a través del proceso de empoderamiento femenino.

Tomado del inglés emporverment, el término denota que el/la sujeto se convierte en agente activo, lo cual es resultado de una acción que varía de acuerdo con cada situación concreta y se concentra en la transformación de las relaciones de poder o del poder como relación social. También puede entenderse como un proceso de superación de la desigualdad de género, y el seguimiento de un proceso por medio del cual las mujeres incrementan su capacidad de configurar sus propias vidas en el entorno. Consiste de igual forma en una evolución en la concientización sobre sí mismas, en su estatus y en la eficacia que 
desarrollan para trascender con sus interacciones sociales (Denman y Cornejo, 1999).

Las manifestaciones que se dan en un proceso de empoderamiento son: 1) sentido de seguridad y visión de un futuro, 2) capacidad de ganarse la vida, 3) capacidad de actuar eficazmente en la esfera pública, 4) mayor poder de tomar decisiones en el hogar, 5) participación en grupos no familiares y usos de grupos de solidaridad como recursos de información y apoyo, 6) movilidad y visibilidad en la comunidad (Denman y Cornejo, 1999).

Si bien vemos que las mujeres que practican la política tienen superados algunos de estos elementos citados por Denman y Cornejo (1999), como es una toma de conciencia sobre lo que desean para su futuro, independencia económica, poder en su espacio privado en decisiones sustanciales y libertad personal en su movilidad, todavía existen aspectos sobre los que hay que incidir y uno de estos es en su capacidad de actuar en política, ya que existen reglas del juego que desequilibran las relaciones de poder en esta esfera.

Su capacidad de actuación en el escenario público queda socavada por 3 factores que proponemos y que llamaremos obstáculos de oportunidad, de acceso y de permanencia:

Obstáculos de oportunidad: Se relacionan con las inseguridades personales que tienen que superar las mujeres para iniciar su camino político, debido a que se autoperciben con una carencia de destrezas, conocimientos y oportunidades que no les permiten transitar en igualdad de condiciones con los hombres.

Obstáculos de entrada: Son los impuestos por la cultura en términos de los estereotipos sobre las esferas de acción y los papeles que deben cumplir las mujeres, aún en el mundo público. Es decir, significa que sus actividades quedan relacionadas regularmente a asuntos de la vida cotidiana.

Obstáculos de permanencia: Se encuentran una vez que las mujeres han logrado entrar en la política y que son las características y dinámicas mismas de este ámbito, con las cuales las 
mujeres no se sienten identificadas y constituyen razones por las que algunas deciden retirarse, pues les dificulta el cumplimiento de sus actividades por actitudes, horarios y espacios que mantienen reglas del juego que no les favorecen.

Estos obstáculos se constituyen en ejes que dificultan la relación mujeres-poder político en México, tal y como lo veremos más adelante con el caso de las chihuahuenses. Al respecto, Bareiro (1998) comenta que en latinoamérica el sexo femenino mantiene escasa participación en las decisiones sobre el conjunto de la sociedad, en la disposición de recursos sociales y en la administración de justicia, pero ha pasado a ser prioritario en la agenda de las mujeres conseguir una ampliación de su ciudadanía a través de la representación, del aumento de su participación en los poderes del estado y las instituciones políticas y sociales.

¿Cómo es la representación de las mujeres en Chihuahua y qué implicaciones tiene esto? En sentido etimológico, la palabra representación proviene del latín representatio, de origen medieval, e indica la imagen, la idea o ambas cosas. Desde el punto de vista conceptual, el politólogo Giovanni Sartori (1998) señala que, en primera instancia, el significado originario de la representación es la actuación en nombre de otro en defensa de sus intereses. Las dos características definitorias de este concepto son, por tanto, a) una sustitución en la que una persona habla y actúa en nombre de otra, b) bajo la condición de hacerlo en interés del representado. Sartori (1998) distingue tres tipos de representación: jurídica, sociológica y política.

1.-Representación jurídica: centra su razón de ser en la idea moderna de mandato. El representante es aquél cuyos actos son imputables a la comunidad que vive bajo la jurisdicción efectiva de este representante. ${ }^{7}$

7 Véase Monedero, Juan Carlos. Representación Política. Diccionario crítico de ciencias sociales. Universidad Complutense de Madrid. Información disponible en www. ucm.es/info/eurotheo/diccionario/.../representacion_politica.htm [consultado el 18 de marzo de 2012]. 
2.-Representación sociológica: hace referencia a la idea de identidad. El representante es aquél en el cual el representado se ve reflejado como en un espejo. El representante pasa a ser un igual o alguien que va defender los intereses del votante por pertenecer a la misma clase social, territorio o practicar una ideología similar.

3.-Representación política: está emparentada con la idea de control y responsabilidad del representante. El elegido debe actuar con responsabilidad respecto de las exigencias de la ciudadanía que lo sostiene, debiendo lograr que se cumplan las exigencias normativas de esa sociedad, de manera que si no son satisfechas le será retirada la confianza.

Aunque Sartori (1998) señala que el concepto de representación es aplicable tanto a la representación jurídica, como al de representación social, existe también un uso sociológico (o existencial) del término que no puede dejarse aparte, ya que tiene acepción diferente. Cuando decimos que alguien o algo son "representativos" estamos expresando una idea de similitud, de identificación o de características compartidas.

Otra autora que aborda el concepto de representación es Hannah F. Pitkin (1985) quien la concibe bajo los siguientes significados:

1.- Representación simbólica: implica hacer presente algo mediante la administración de mecanismos psicológicos o emotivos. Se fundamenta en un sistema de valores, creencias y actitudes, y esto es lógicamente un aspecto subjetivo. De manera que conciente o inconcientemente estamos autorizando una representación de acuerdo a nuestras decisiones.

2.-Representación política: hace alusión a una relación de compromisos de hechos y valores, fines y medios. Involucra personas, grupos e instituciones los cuales se vinculan a una estructura de poder para actuar en la resolución de conflictos en beneficio de sus representados. 
Cabe señalar que el concepto de representación política concierne a la democracia representativa, los procesos electorales, partidos políticos, candidatos e instituciones, etcétera. También se encuentra apegado a la cuestión institucional de representación de intereses y ve a la política como una actividad orientada en forma ideológica a la toma de decisiones para el alcance de ciertos objetivos. En lo que respecta a la representación simbólica, Pitkin (1985: 106) sugiere que se trata de creencias irracionales tomadas del entorno cultural al que se pertenece. Las relacionadas con el mundo femenino implican según el imaginario social tradicional, que la política pertenece al mundo público-masculino y que si bien ellas están presentes no es su espacio natural de adscripción, por esa razón, es normal observarlas como sujetos no empoderados que deben atenerse a las reglas preexistentes, sin importar que no sean en su beneficio político.

Una vez entendido el concepto de representación y sus distintas acepciones, ahora hablemos sobre política. La utilización del término ganó popularidad en el siglo $V$ a.C., cuando Aristóteles desarrolló su obra titulada justamente La política. La cual proviene de la palabra griega polis; y como señala Sartori (1984:208) “...está ligada indisolublemente al espacio de la polis..." cuyo significado hace alusión a las ciudades griegas que formaban los estados donde el gobierno era parcialmente democrático. Cabe señalar, que es en esta cultura donde intenta formalizarse esta necesidad humana de organizar la vida social y los gobiernos desde tiempos ancestrales hasta los modernos.

Para la mayoría de los pensadores tanto clásicos y contemporáneos como Bobbio, Dalh, Duverger, etcétera, la política es el estudio de las luchas por el poder entre los hombres, por lo que de entrada queda señalada como un espacio de y para los hombres y establecida en el espacio público, es decir, la esfera de la producción; quedando así las mujeres confinadas a la esfera de la reproducción debido a su naturaleza humana. De manera que las relaciones de subordinación prevalecieron dejándolas excluidas de la libertad política, y aún con la incorporación de ciertos derechos no se ha alterado esta estructura. Dicha exclusión se atribuyó a su inhabilidad para trascender su subordinación biológica y económica del espacio doméstico, por lo tanto, la distinción entre 
dominantes y dominadas se volvió algo específico del sexo, y la política por definición se convirtió en un mundo en el que los hombres actuaban con ventajas preestablecidas (Jones, 1992).

De acuerdo con García Cosío (2012: 15) se han dado casos en donde alguna mujer ha traspasado los códigos de lo que "debe hacer una buena mujer" y la respuesta ha sido de rechazo y expulsión de la política cuando alguno ha sentido "invadido su espacio". Según García Cosío (2012), a las mujeres aún se les ubica en un plano más emocional y de actividad doméstica, lo que supuestamente limita su participación política en el ámbito público (ver cuadro 1):

Cuadro 1: Comparativo de aspectos que limitan la participación y representación del hombre y la mujer en el ámbito público

\begin{tabular}{|l|l|}
\multicolumn{1}{|c|}{ HOMBRE } & \multicolumn{1}{c|}{ MUJER } \\
\hline Mundo laboral & Mundo doméstico \\
\hline Cívico y político & \multicolumn{1}{c|}{-} \\
\hline Trabajo remunerado & Ama de casa, madre y esposa \\
\hline Autonomía y ciudadanía & Ser para otros \\
\hline Proveedor económico (dinero) & Proveedora emocional (Amor) \\
\hline Poder público (mandar) & Poder en la intimidad (obedecer) \\
\hline Individuo & Genérico \\
\hline Nombrar, visible & Innombrable, invisible \\
\hline
\end{tabular}

Fuente: García Cosío María lleana, "Género y participación política: el caso de Rosario Robles". Revista alcaldes de México, mujeres políticas, pp. 14-15, No. 25, marzo 2012.

Desde el punto de vista de la representación simbólica, existen aspectos subjetivos que inciden en la formación de las mujeres como seres estereotipados a quienes supuestamente no les interesa el poder o le temen. Lo anterior está claramente expuesto por Mabel Burín (1996) y su idea sobre el llamado Techo de Cristal. ${ }^{8}$ Este techo de

8 Según esta especialista en psicología clínica y estudios de género se denomina así a una superficie superior invisible en la carrera laboral de las mujeres, difícil de traspasar, que nos impide seguir avanzando. Su carácter de invisibilidad viene dado por el hecho de que no existen leyes ni dispositivos sociales establecidos ni códigos visibles que impongan a las mujeres semejante limitación, sino que está construido sobre la base de otros rasgos que por su invisibilidad son difíciles de detectar. 
cristal que impide a las mujeres alcanzar las metas profesionales para las que están preparadas parece invisible. Sin embargo, las estadísticas demuestran que existe; es decir, es un término enigmático, secreto, indetectable, pero cuyo resultado es cuantificable y real: la no existencia de mujeres en los vértices jerárquicos de las organizaciones y en los espacios públicos como ya se describía al principio.

Siguiendo a Mabel Burín (1996) en el asunto de los estereotipos y concepto del techo de cristal; se formulan algunas representaciones sociales de la siguiente manera: "las mujeres temen ocupar posiciones de poder", "a las mujeres no les interesa ocupar puestos de responsabilidad", "las mujeres no pueden afrontar situaciones difíciles que requieran autoridad y poder".

Estos estereotipos tienen múltiples incidencias: por una parte, convierten a las mujeres en "no-elegibles" para puestos que requieran autoridad y ejercicio del poder. Por otra, hay mujeres que asumen este estereotipo interiorizándolo, repitiéndolo casi sin cuestionarlo y como si fuera resultado de elecciones propias. También es cierto que hay grupos de mujeres más innovadoras que, admitiendo el conflicto, lo enfrentan con recursos variados cada vez que ocupan tales puestos de trabajo (García Cosío, 2012).

Es conocido que las mujeres profesionales, incluyendo las que se dedican a la política, duplican jornadas laborales para compaginar su trabajo fuera de casa con las tareas domésticas, a menudo de forma unilateral. El entrenamiento predominante de las mujeres en los vínculos humanos con predominio de la afectividad puede entrar en contraposición ( $\mathrm{y}$ de hecho entra en infinidad de ocasiones) con ese mundo del trabajo masculino, donde los vínculos humanos se caracterizarían por un máximo de racionalidad y con afectos puestos en juego mediante emociones frías: distancia afectiva, indiferencia, etcétera. Esto es lo que se conoce en el mundo de la política y el ejercicio del poder como la masculinización de las actividades.

En opinión de especialistas como Mabel Burín, el trabajo de muchas mujeres en espacios tradicionalmente masculinos, es observado de forma más inquisitiva que el de sus compañeros varones, es decir, se les exige un nivel de excelencia. Las reglas del juego o las relaciones 
de poder que caracterizan la política institucional en México son otra limitante a las que comúnmente se tienen que enfrentar las mujeres para alcanzar las posiciones importantes dentro de la política o la administración pública, también tienen que hacer frente a prácticas en las que mediante acuerdos con varones se ven forzadas a ceder su lugar a éstos, como sucedió a dos días de haber iniciado la LXI Legislatura federal, cuando ocho diputadas con suplentes varones "solicitaron" licencia para dejarles el cargo: ${ }^{9}$

Las legisladoras, todas ellas plurinominales, recibieron el nombre de diputadas juanitas y, pese a las críticas públicas, la mayoría obtuvo licencia para separarse de sus puestos. Cuatro de ellas militan en el Partido Verde Ecologista (Carolina García, María Ivette Ezeta, Laura Elena Ledesma y Kattia Garza), dos en el PRI (Yulma Rocha y Ana María Rojas), una en el PT (Anel Nava) y una en el PRD (Olga Luz Espinosa).

La inclusión de las mujeres en el campo político incluye romper barreras que provienen de la familia, la educación patriarcal y la cultura social discriminatoria (González, 2008). Existen aspectos contra los que tienen que lidiar en su integración política al tener que sobrepasar la discriminación de género y las fuerzas que dirigen la vida institucional en asuntos tan comunes como son la designación de comisiones de mayor o menor importancia, las que se relacionan con cuestiones asistenciales, espacios de negociación, horarios y hasta las jornadas de trabajo a las que están expuestas, como lo veremos más adelante.

9 A este acto se le conoció en México como el de "las juanitas", en alusión al caso de Rafael Acosta Ángeles “Juanito”, quien en 2009 llegó a la jefatura delegacional en Iztapalapa como candidato del Partido del Trabajo, pero solicitó licencia para dejar el cargo en manos de la perredista Clara Brugada. Fuente www.mexico.cnn.com [consultado el 29 de abril de 2012]. 


\section{Mujeres en la política chibuabuense}

El sistema de género se encuentra imbricado en todas las prácticas políticas, de manera que se considera importante tomar en cuenta dos cuestiones al respecto: 1) $\mathrm{El}$ análisis del género en el ámbito político y 2) Evitar restringir su estudio en el contexto institucional (espacio de las instituciones y partidos políticos), con el propósito de evidenciar y hacer patente las ausencias de las mujeres, al incluir otros escenarios que trasciendan una definición restrictiva de la política (Astelarra, 1990).

La propuesta que aquí se presenta tiene un interés genuino para contribuir al conocimiento en los fenómenos de las ciencias sociales en particular de la ciencia política, así como ofrecer una evidencia empírica sobre la condición de subordinación y exclusión del género femenino en los puestos de toma de decisión y de poder.

En este sentido, es importante reconocer que los trabajos de investigación sobre mujeres y teoría política son necesarios en esta región, a fin de que puedan contribuir a enriquecer esta línea de investigación. La siguiente gráfica demuestra que la producción académica y de investigación de universidades como la de Ciudad Juárez (UACJ), de Chihuahua (UACH) y Colegio de la Frontera Norte (COLEF sede Juárez) se caracteriza por ser escasa.

Gráfica 1: Líneas de investigación sobre temas de género desarrolladas de 1990 a 2012, en las Instituciones Superiores más importantes del norte de Chihuahua (UACJ, COLEF, UACH)

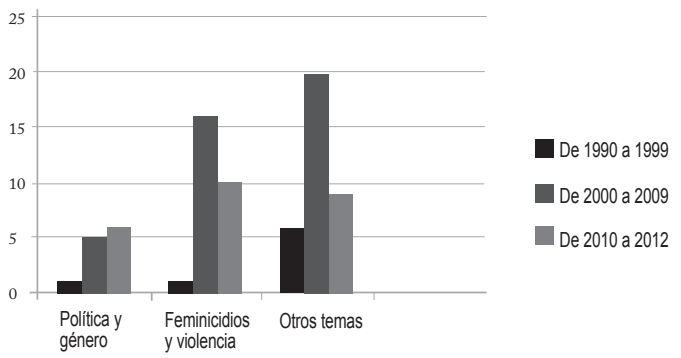

Fuente: elaboración e investigación propia 
El interés de la presente investigación se relaciona con la contribución al conocimiento de los fenómenos políticos del estado de Chihuahua y se tomará como punto de partida la participación política. Es en la práctica donde se hace visible la situación de la mujer en la política, por lo que a través de algunos datos que se presentan, se muestra su condición en esta entidad.

Cuadro 2: Partidos políticos que conforman

el Congreso del Estado de Chihuahua

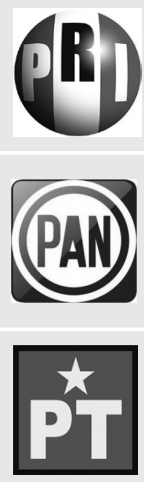

20 miembros

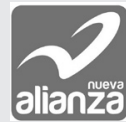

4 miembros

6 miembros

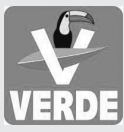

1 miembro

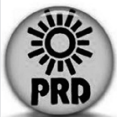

1 miembro

De acuerdo con datos obtenidos del Instituto Nacional de Estadística y Geografía (INEGI),10 la política es una esfera de participación asimétrica entre hombres y mujeres. El ámbito de la participación y representación en el estado de Chihuahua es efímera, pues sólo representa el 4.47\%; es decir, sólo tres mujeres presidentas municipales de un total de 67 municipios que conforman el estado más grande del país. No se reportan mujeres síndicos, aunque en el 2008 se documentó un $43.8 \%$ de regidoras en los distintos municipios.

Los regidores y regidoras son elegidos cada 3 años en elecciones locales a través del voto ciudadano. La Ley Electoral del Estado de Chihuahua señala en su artículo 78 , inciso 4 , que “...las candidaturas a miembros de los ayuntamientos se registrarán en planillas que esta-

10 Fuente INEGI (2008). 
rán integradas cada una por un presidente municipal y un número de regidores que determine el Código Municipal, todos con su respectivo suplente ante la Asamblea Municipal correspondiente...”.

Actualmente (periodo 2010-2013) en Ciudad Juárez se reporta la participación de un 50\% de mujeres como regidoras (ver cuadro 3). Si bien este es un dato significativo y pareciera un signo de equidad, se cuestiona la existencia de un empoderamiento real por las posiciones que ocupan y la toma de decisiones en cuestiones no trascendentales. Por lo regular, se ubican en comisiones donde su participación no tiene un peso definitivo en la toma de decisiones, o bien, no son áreas estratégicas y se encuentran ligadas a asuntos más cotidianos y domésticos, mientras que los varones suelen dirigir comisiones donde se toman decisiones primordiales de gobierno.

En esta ciudad fronteriza las regidoras coordinan las áreas de $\mathrm{Tu}-$ rismo, Familia y asistencia social, Nomenclatura y Monumentos, Trabajo y Previsión Social, Salud pública, Obras públicas, Desarrollo Urbano y Asentamientos humanos; mientras que los varones ocupan las comisiones de Hacienda, Gobernación, Desarrollo Social, Centros comunitarios, Seguridad pública, etcétera.

Cuadro 3: Regidores(as) que integran el Ayuntamiento de Juárez (2010-2013)

\begin{tabular}{|c|c|c|c|c|c|c|}
\hline $\begin{array}{l}\text { Partido/ } \\
\text { Coalición }\end{array}$ & $\begin{array}{c}\text { Total de } \\
\text { espacios en } \\
\text { el cabildo }\end{array}$ & 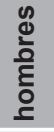 & $\%$ & $\frac{\mathscr{d}}{\frac{0}{3}}$ & $\%$ & $\begin{array}{c}\text { Presencia } \\
\text { de mujeres } \\
\text { en el cabildo }\end{array}$ \\
\hline PRI & 9 & 4 & $22.2 \%$ & 5 & $27.7 \%$ & \multirow{5}{*}{$50 \%$} \\
\hline PAN & 7 & 4 & $22.2 \%$ & 3 & $16.6 \%$ & \\
\hline PRD & 1 & 0 & $0 \%$ & 1 & $5.5 \%$ & \\
\hline PANAL & 1 & 1 & $5.5 \%$ & 0 & $0 \%$ & \\
\hline Total & 18 & 9 & $50 \%$ & 9 & $50 \%$ & \\
\hline
\end{tabular}

Fuente: Elaboración propia con la información obtenida de las entrevistas realizadas para efectos de esta investigación. 
En el caso del Poder Legislativo del Estado de Chihuahua, el congreso $^{11}$ está integrado por 33 diputados (2010-2013), de los cuales 22 son elegidos por el principio de mayoría relativa y el resto por el de representación proporcional. Por cada diputado propietario se elige un suplente. Los partidos políticos que tienen representación en el Congreso del Estado y que han conformado sus grupos parlamentarios son: el PRI, el PAN, el PANAL, el PRD y el PT (ver cuadro 4).

Cuadro 4: LXIII Legislatura del estado de Chihuahua (2010-2013)12

\begin{tabular}{|c|c|c|c|c|c|c|}
\hline $\begin{array}{l}\text { Partido/ } \\
\text { Coalición }\end{array}$ & $\begin{array}{l}\text { Total de } \\
\text { Escaños } \\
\text { ganados }\end{array}$ & $\begin{array}{l}\text { d } \\
\text { 을 } \\
\text { 욷 }\end{array}$ & $\%$ & 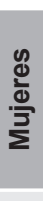 & $\%$ & $\begin{array}{l}\text { Presencia de } \\
\text { mujeres en el } \\
\text { Poder } \\
\text { Legislativo }\end{array}$ \\
\hline PRI & 19 & 17 & 89.4 & 2 & 10.5 & \multirow{6}{*}{$21.20 \%$} \\
\hline PAN & 6 & 4 & 66.6 & 2 & 33.3 & \\
\hline PANAL & 4 & 3 & 75 & 1 & 25 & \\
\hline PRD & 1 & 1 & 100 & 0 & 0 & \\
\hline PVEM & 2 & 0 & 0 & 2 & 100 & \\
\hline PT & 1 & 1 & 100 & 0 & 0 & \\
\hline Total & 33 & 26 & 78.7 & 7 & 21.2 & \\
\hline
\end{tabular}

Fuente: Elaboración propia a partir de la información proporcionada por el Congreso del Estado de Chihuahua, respecto a la conformación de la LXIII legislatura.

De los 33 diputados, sólo siete son mujeres; dos de ellas elegidas por el principio de mayoría relativa, ${ }^{13}$ las otras cinco llegaron por el

11 El Congreso se integra por representantes del pueblo de Chihuahua, electos como diputados en su totalidad cada tres años.

12 Los(as) diputados(as) locales del estado de Chihuahua fueron elegidos/as constitucionalmente el 4 de julio de 2010 y tomaron posesión el 1 10 de octubre del mismo año.

13 La Ley Electoral del Estado de Chihuahua establece una cuota de género que no sobrepase el $70 \%$ de un mismo sexo. 
principio de representación proporcional o plurinominales $;{ }^{14}$ es decir, la proporción de mujeres con respecto a sus pares masculinos es de apenas un $21.20 \%$ (ver cuadro 5).

Cuadro 5: LXIII Legislatura del estado de Chihuahua (2010-2013) ${ }^{15}$

\begin{tabular}{|c|c|c|c|c|c|c|}
\hline $\begin{array}{l}\text { Partido/ } \\
\text { Coalición }\end{array}$ & $\begin{array}{l}\text { Total de } \\
\text { escaños } \\
\text { ganados }\end{array}$ & 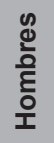 & $\%$ & 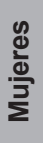 & $\%$ & $\begin{array}{c}\text { Presencia de } \\
\text { mujeres en el } \\
\text { Poder Legislativo }\end{array}$ \\
\hline PRI & 19 & 17 & 89.4 & 2 & 10.5 & \multirow{7}{*}{$21.20 \%$} \\
\hline PAN & 6 & 4 & 66.6 & 2 & 33.3 & \\
\hline PANAL & 4 & 3 & 75 & 1 & 25 & \\
\hline PRD & 1 & 1 & 100 & 0 & 0 & \\
\hline PVEM & 2 & 0 & 0 & 2 & 100 & \\
\hline PT & 1 & 1 & 100 & 0 & 0 & \\
\hline Total & 33 & 26 & 78.7 & 7 & 21.2 & \\
\hline
\end{tabular}

Fuente: Elaboración propia a partir de la información proporcionada por el Congreso del Estado de Chihuahua, respecto a la conformación de la LXIII legislatura.

Estos datos muestran la presencia de la mujer en la esfera pública chihuahuense, aunque no con un rol destacado, ni con un empoderamiento efectivo. No obstante la escasa presencia femenina, se descarta el estereotipo de que a las mujeres no les interesa la política o la interacción en el espacio público-institucional, ya que nos muestra que

14 La Constitución Política del Estado de Chihuahua señala que para la asignación de diputados electos por el principio de representación proporcional, cada partido político deberá registrar una lista de seis fórmulas de candidatos y suplentes, la cual no podrá contener entre propietarios y suplentes más del 70\% de candidatos de un mismo género.

15 Los(as) diputados(as) locales del Estado de Chihuahua fueron elegidos/as constitucionalmente el 4 de julio de 2010 y tomaron posesión el $1^{\circ}$ de octubre del mismo año. 
están ahí, aun con la brecha de inequidad que experimentan en una lucha contracorriente.

Los primeros resultados del acercamiento que se tuvo con algunas diputadas de la LXIII Legislatura (2010-2013) y regidoras del Municipio de Juárez (2010-2013), a través de entrevistas que se realizaron para conocer las prácticas políticas que deben desarrollar para sobrevivir en la esfera pública, fueron los siguientes: ${ }^{16}$

Se encontró que la familia tiene un gran peso en la toma de decisiones de las mujeres para su involucramiento en política. Argumentó la mayoría de ellas haber iniciado con una conciencia social durante su niñez y adolescencia, por lo que participaron en actividades de beneficio social, activismo escolar, comunitario o partidista con total apoyo de sus seres cercanos. La mayor parte de las entrevistadas mencionó al padre como uno de los principales inspiradores de su vinculación a lo público, ya que algunos de ellos eran militantes de partidos políticos, funcionarios públicos o tenían un marcado interés en los procesos electorales, principalmente el ejercicio del voto. De esta manera, se puede decir que la familia constituye un eje clave de apoyo en su toma de decisión para participar en la esfera pública.

De igual manera, se encuentra que las que tienen pareja cuentan con su respaldo incondicional, ya que mencionaron que de no ser así "difícilmente podríamos dedicarnos a las actividades públicas". Sin embargo, en el caso de las que tienen hijos menores de edad, comentaron que "ha sido muy difícil compaginar la actividad política con la maternidad". Por su parte, las que tienen hijos de mayor edad, consideraron que su trabajo era más fácil porque no era tan demandante la atención que había que darles. Con relación al ámbito doméstico, todas dijeron que siguen ocupándose de las decisiones de casa y ninguna

16 Se aplicó una entrevista semiestructurada bajo tres aspectos principales: 1) Profesionales, 2) Personales y 3) Opiniones sobre la condición de género. El primero contempló preguntas como escolaridad, trayectoria estudiantil, participación social y política durante esta época. Se preguntó sobre sus obstáculos de partida, entrada y permanencia en el ámbito político. El segundo aspecto averiguó vida familiar y relación de pareja y administración de su tiempo y recursos económicos. Finalmente, se recogieron sus opiniones sobre las condicionantes del género en su vida personal, familiar y política. 
mencionó haber dejado este rol, lo cual demuestra que no existe aún un desligue del marcaje cultural.

Se encontró que la preparación educativa y la capacitación profesional son determinantes en su estatus laboral y político, ya que sólo una de las entrevistadas no cuenta con carrera profesional terminada, pero es estudiante universitaria, todas las demás tienen licenciaturas y estudios de posgrado. Esta condición es fundamental para entrar en competencia con sus pares políticos, sin embargo, esto "no nos otorga un poder de facto, ni un reconocimiento, ya que tenemos que demostrar día con día nuestras capacidades" ante sus compañeros para tener aceptación a su esfuerzo laboral, según manifestaron.

Las entrevistadas comentaron que su principal motivación para participar en política "era poder ayudar a los demás u otorgar protección, pero sobre todo su interés por la comunidad y la resolución de sus problemas", es decir, su visión es apegada todavía a la idea de la labor que la mujer hace en casa y una actitud de servicio a los otros(as). Sin embargo, esto no hay que verlo como una desventaja, pues las sensibiliza ante los problemas sociales, lo que se convierte en una cualidad que las hace diferentes respecto a sus pares masculinos.

La relación con sus respectivos partidos políticos es otro elemento clave en su vida pública. Expresaron que se basa en un vínculo de lealtad conformado por el tiempo de militancia, ya que la mayoría dijo haberse sostenido activa por años y haber desempeñado algunos puestos al interior de éstos, realizado algún tipo de participación de apoyo propagandístico o siendo candidatas. Incluso una mayoría, alguna vez fue suplente a un cargo de elección popular. Al respecto de este punto, refirieron "las suplencias no son negativas para el desarrollo de las mujeres, al contrario, son oportunidades de aprendizaje y preparación”.

Por ejemplo, dijeron que la Ley de cuotas de género, lejos de ser un obstáculo por la limitación que tienen en los porcentajes de representación y participación, por ser manejado en múltiples ocasiones de manera desventajosa para ellas, lo visualizaron como "una oportunidad de ascenso y permanencia en el poder, ya que si no fuera obligatorio para los partidos políticos tener candidatas, difícilmente lo harían". Otro elemento que habría que señalar es el conflicto de intereses que se les 
presenta en ocasiones. En el caso de que pretendan sacar adelante una agenda de trabajo, ésta no debe entrar en debate con el partido y sus intereses institucionales, puesto que se ven obligadas a respetarlos, aun por encima de sus propias decisiones.

Al pedirles comentarios sobre su percepción de discriminación, ninguna de las entrevistadas se consideró discriminada, pero reconocieron ciertas prácticas que las colocan en desventajas respecto del ejercicio político de sus pares masculinos, por ejemplo "los horarios y espacios de trabajo no siempre resultan cómodos" para su condición de mujer, ya que en ocasiones ciertos acuerdos se toman en lugares no adecuados para este propósito y esto las limita en su asistencia, pues no pueden hacerlo o suelen no ser invitadas. Otro de los casos es el no poder renunciar al rol de género, lo que les impide mantener el mismo ritmo de trabajo que sus compañeros, al realizar jornadas dobles o triples. Si bien, manifestaron que la relación con sus pares políticos masculinos es bajo un ambiente de respeto y no refirieron abiertamente ser discriminadas, se observa que son relegadas y esto demuestra un ambiente de inequidad.

\section{Conclusiones}

En este documento hacemos un acercamiento que nos permitió explicar a través del análisis de las opiniones, experiencias y trayectorias de algunas mujeres que participan en la práctica política institucional del Estado de Chihuahua, la problemática de la representación simbólica y política del sexo femenino, así como su falta de empoderamiento.

Si lo vemos en los términos señalados por Pitkin (1985), encontramos en la representación simbólica que lo considerado como femenino sigue teniendo peso en el campo político, ya que está fundamentado en la creencia estricta de la división sexual del trabajo (DST), bajo una idea arraigada de los espacios y contextos que las mujeres deben ocupar aún en los quehaceres políticos, tal y como lo hemos visto en los hallazgos de esta investigación: ellas suelen ocuparse de actividades relacionadas al bienestar social y es difícil que sean sujetos de actividades relacionadas a la toma de decisiones de peso en la estructura de 
poder. Se observa que la cultura sigue presente en las concepciones del deber ser de las mujeres. Son relegadas a roles tradicionales y si bien las mujeres tienen conciencia de esto, no hacen esfuerzo por cambiar esta situación. Ellas se muestran dispuestas a servir a los intereses partidistas por dos razones principales: una es su sentido de lealtad y la otra por preservar un estatus de privilegio político, porque saben que sólo así podrán seguir vigentes en su trayectoria.

Respecto a la representación política, las mujeres que llegan a ocupar algún puesto de representación popular lo hacen sujetas al cumplimiento estricto de valores, fines y medios de los partidos políticos y no de sus propios intereses políticos. La representación política de mujeres en el estado de Chihuahua se caracteriza por ser insuficiente, es decir, el número de mujeres en la política no es proporcional con respecto a sus pares masculinos. Dicha situación genera exclusión e inequidad para las políticas por los escasos espacios que ocupan. Sin embargo, su ingreso en este escenario tampoco garantiza un cambio debido a que se les asignan comisiones y actividades de poca importancia y trascendencia pública. Asimismo, existen contradicciones hacia el interior de los distintos partidos políticos, ya que a pesar de que ellas suelen ser muy activas en su desempeño, siguen siendo discriminadas por las pocas oportunidades que les ofrecen. Solamente se cumple con lo establecido por la ley y no ceden espacios para estimular la participación femenina, ya que esto implicaría pérdida de espacios para los que detentan el poder.

En términos de representación política, la equidad de género todavía está lejos de alcanzarse, tal y como se observa en los datos ofrecidos. Esta subrepresentación no sólo la padecen las mujeres en los números, también se observa una representación simbólica estereotipada en las relaciones de poder que se entablan al interior del espacio político, en el cual se siguen reproduciendo los esquemas culturales tradicionales. De manera que se encuentra una relación directa entre la representación simbólica y política, la primera influye directamente a la segunda y es determinante en la creación e interacción de los contextos políticos.

En este sentido, el empoderamiento de las mujeres no es factible y enfrenta obstáculos y retos pendientes, mientras que ellas no sean 
vistas como sujetos con capacidades iguales por sus pares masculinos, se respeten los horarios y espacios de trabajo, y se les incluya en la toma de decisiones estratégicas, la cultura democrática no tendrá un avance real.

Mientras que ellas no tengan una toma de conciencia de la discriminación de que son objeto, pues si bien se desarrollan como mujeres que han escalado al poder, éste es un lugar hostil para su desarrollo político y personal. Se hace necesario que se cultive una conciencia de género que les permita unirse y fortalecerse ante las adversidades que enfrentan, para que sea factible un avance como grupo político que integre los intereses femeninos en las agendas de gobierno.

Como lo señala Yolanda Morales (2012) regidora de Ciudad Juárez: "El mayor obstáculo es que no existe una conciencia plena entre las mujeres sobre su derecho a participar activamente en la política y puestos de poder; y las pocas mujeres que se encuentran en el poder consideran que entre el género femenino no hay solidaridad y apoyo para las que se encuentran en posiciones estratégicas y jerárquicas importantes".

Finalmente, queda decir que este primer acercamiento ha creado nuevas interrogantes que nos permitirán seguir avanzando en una investigación más profunda y que maneje otras vertientes. Sería significativo centrarse en la vida interna de los partidos políticos porque ellos son los responsables directos de integrar, dirigir y potencializar las trayectorias de las mujeres que militan en ellos. Conociendo los adentros de sus estructuras, ideologías, plataformas e historias, será posible responder a algunos de los pendientes que quedan: nuestra propuesta es observar bajo la mirada de los partidos políticos, con el propósito de identificar y analizar las posturas que guardan hacia los sujetos femeninos, a los cuales sólo han dejado saldos negativos. 


\section{Fuentes}

Bareiro, Line. "Las recién llegadas. Mujer y participación política". En Bareiro, Line y Soto Clyde (coords.) Sola no basta. Mecanismos para mejorar la participación politica de las mujeres. CDE-Fundación Friedrich Ebert, Asunción, Paraguay 1998. Documento en línea. http://www.iidh.ed.cr/comunidades/derechosmujer/docs/ dm_documentospub/Estudios\%20Basicos\%204/8.\%20Las\%20 recien\%20llegadas.pdf [Consultado el 16 de abril de 2012]

Burín, Mabel y Emilce D. Bleichmar. Género, psicoanálisis, subjetividad. Buenos Aires, Paidós. 1996.

Butler, Judith. Cuerpos que importan. Sobre los limites materiales y discursivos del sexo. Paidós. Buenos Aires. 2002.

CNN México. "Juanitas, una estrategia política para evadir la ley". Noticia del lunes 5 de diciembre de 2011. Disponible en www. mexico.cnn.com

Denman, Catalina y Elsa Cornejo, et. al. "Poder y empoderamiento de las mujeres". En Región y Sociedad. Vol. XI No. 18, 1999, pp. 189-197. Documento en línea http://lanic.utexas.edu/project/ etext/colson/18/18_8.pdf [Consultado el 29 de abril de 2012].

El género en disputa. El feminismo y la subversión de la identidad. Paidós. México, D.F. 2001.

Monedero, Juan Carlos. "Representación política". En Diccionario Critico de las Ciencias Sociales. Universidad Complutense de Madrid. En línea www.ucm.es/info/eurotheo/diccionario/.../ representacion_politica.htm [consultado el 15 de octubre de 2012].

García Cossío, María Ileana. "Más presencia pero...¿más poder?”. Revista Alcaldes de México, no. 25, marzo 2012.

González Marín, María Luisa. "Mitos y realidades sobre la participación política de las mujeres". En González Marín, María Luisa y Patricia Rodríguez (Coords.) Limites y desigualdades en el empoderamiento de las mujeres en el PAN, PRI y PRD. Porrúa-UAMI-UNAM. México, D.F., 2008, pp. 19-32. http://redalyc.uaemex. 
$\mathrm{mx} / \mathrm{redalyc} / \mathrm{html} / 267 / 26700119 / 26700119 . \mathrm{html}$ [Consultado el 29 de abril de 2012].

Jones, Kathtleen B. "Hacia una revisión de la política". en Revista Política y Cultura, Otoño, No. 1. UAM-X. México, D.F. 1992, pp. 277-298. Documento en Línea.

Lagarde, Marcela. Democracia Genérica. Red Latinoamericana de Educación Popular Entre Mujeres. REPEM-México y Mujeres para el Diálogo. México, 1994.

Lamas, Martha. Feminismo. Transmisiones y retransmisiones. Taurus. México, D.F., 2006.

Pitkin, Hannah. El concepto de Representación. Centro de Estudios Constitucionales, Madrid. 1985.

Sartori, Giovanni. "En defensa de la representación política". Conferencia dictada en el Congreso de los diputados españoles con motivo del vigésimo aniversario de la Constitución de 1978. Revista claves de razón práctica. No. 91, 1999.

--- La Política-Lógica y Método en las Ciencias Sociales. FCE. Mexico, D.F. 1984.

\section{Páginas electrónicas}

Congreso Estatal del Estado de Chihuahua. Documento en línea www. congresochihuahua.gob, $\mathrm{mx} /$ diputados/partidos.php. [Consultado el 17 de marzo de 2012.

Diccionario crítico de ciencias sociales. Universidad Complutense de Madrid. Disponible en www.ucm.es/info/eurotheo/diccionario/.../ representacion_politica.htm [consultado el 18 de marzo de 2012].

Instituto Nacional de las Mujeres. Documento en línea www.inmujeres. gob.mx [Consultado el 18 de marzo de 2012].

Instituto Federal Electoral. Documento en línea. www. http://www. ife.org.mx/portal/site/ifev2 [Consultado el 20 de abril de 2012].

Ley Electoral del Estado de Chihuahua. http://www.chihuahua. gob.mx/atach $2 /$ codesoypc/uploads/Lecturas $\% 20$ de $\% 20$ Pol\%C3\%ADtica\%20Social/Normativa/\%C3\%81 mbito\%20 
estatal/Ley\%20electoral\%20de1\%20estado\%20de\%20Chihuahua. pdf [Consultado el 20 de abril de 2012].

Periódico Los Andes, documento en línea http://www.losandes.com. ar/notas/2010/11/1/presidentas-america-latina-524571.asp [Consultado el 25 de marzo de 2012].

Periódico El universal, documento en línea, http://yopolitico. redpolitica.mx/notas/?p=1359 [consultado el 25 de marzo de 2012].

Programa de Naciones Unidas para el Desarrollo(PNUD).Documento en línea, www.undp.org.mx/spip.php?page=imprime\&id_ article $=1758$ [Consultado 31 de octubre de 2012].

World Press. Documento en línea. www.http://informacionpolitica. wordpress.com/category/legislatura [Consultado el 20 de abril de 2012].

\section{Entrevistas}

\section{Regidoras}

Contreras, Xochitl. PAN. 3 de abril de 2012.

Gaytán, Josefina. PAN. 3 de abril de 2012.

Morales, Alma Yolanda. PRI. Ex diputada local. 3 de abril de 2012.

Ruiz Ríos, Paola Berenice. 12 de abril de 2012.

Tarín, Irma. PRI. 2 de abril de 2012.

\section{Diputadas}

Aguilera, Liz. PRI. 10 de abril de 2012.

Gómez Licón, Ana. 9 de abril de 2012.

Martínez Bernal, Inés Aurora. PAN. 10 de abril de 2012. 